\title{
ON CERTAIN AGGREGATES OF DETERMINANT MINORS*
}

\author{
BY
}

W. H. METZLER

\section{Introduction and General Notations.}

1. Since the announcement $\uparrow$ by KRONECKER, in 1882 , of his now well-known theorem regarding linear relations between the minors of an axisymmetric determinant various papers $\ddagger$ have appeared treating of the subject. Dr. MuIr in his paper of 1888 showed that a similar relation exists between the minors of a centrosymmetric determinant and in his paper of 1900 he gives the following two theorems :

Theonem $\mathrm{A}$ : If $\mu$ and $\nu$ be any integers, $\mu$ being the less, taken from the series $n, n+1, n+2, \ldots, 2 n$ and $a, \beta, \gamma, \ldots, \omega$ be what the series becomes when $\mu$ is removed, and $a, \beta, \gamma, \ldots, \psi$ what it becomes when both are removed; then in connection with any even-ordered determinant ||$_{12}^{12} \cdots 2 n \mid$ we have

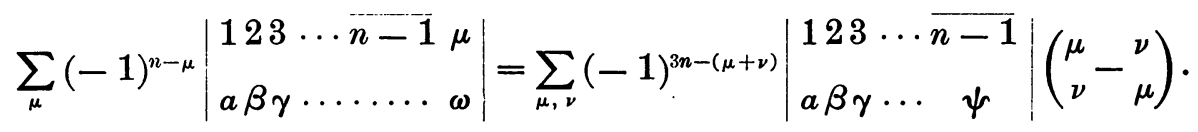

Theorem B: If the symbol

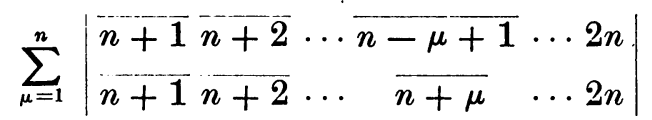

stand for the sum of the $n$ determinants whose column-indices are in every case

* Presented to the Society A pril 27, 1901. Received for publication August 31, 1901.

$\dagger$ Kron hcker, L., Die Subdeterminanten symmetricher Systeme, B erli in er Berich te, 1882.

$\ddagger$ RUNGE, C., Die linearen Relationen zwischen den verschiedenen Subdeterminanten symmetricher Systeme, Crelle's Journal, vol. 93.

Мкнмке, R., Bemerkung über die Subdeterminanten symmetricher Systeme, Mathematische Annalen, vol. 26.

Schendel, L., Die Kronecker'sche Subdeterminantensatz, Zeitschrift für Mathematik und Physik, vol. 32.

MuIr, T., On Vanishing Aggregates of Determinants, Proceedings of the Royal Sooiety of Edinburgh, 1888. On Certain Aggregates of Determinant Minors, ibid., 1900.

White, H. S.. Kronecker's Linear Relation among Minors of a Symmetric Determinant, Bulletin of the American Mathematical Society, 1896. 
$n+1, n+2, \ldots, 2 n$ and whose row-indices are the same except that for one of them there has been substituted its defect from $2 n+1$; and if

$$
\left|\begin{array}{ccccc}
\overline{n+1} & n+2 & \ldots & \overline{n-\mu+1} & \ldots 2 n \\
\overline{n+1} & \overline{n+2} & \ldots & \overline{n+\mu} & \ldots 2 n
\end{array}\right|
$$

be taken to indicate that in the determinant

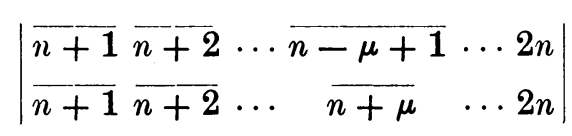

each element ${ }_{\beta}^{a}$ of the $\mu$ th row is to be diminished by the element ${ }_{2 n+1-\beta}^{2 n+1-a}$; then,

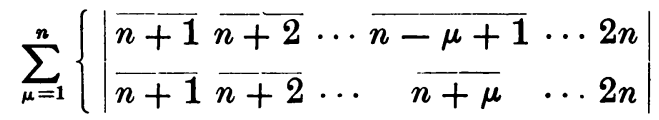

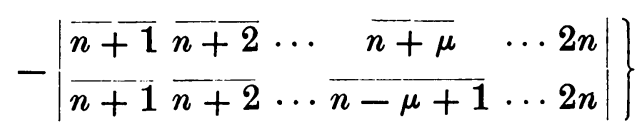

$$
\begin{aligned}
& =\sum_{\mu=1}^{n}\left|\begin{array}{lllll}
\overline{n+1} & \overline{n+2} & \ldots & \overline{n-\mu+1^{\prime}} & \ldots 2 n \\
\overline{n+1} & \overline{n+2} & \ldots & \overline{n+\mu} & \ldots 2 n
\end{array}\right| \text {. }
\end{aligned}
$$

If the determinant $\left|\begin{array}{ll}12 \cdots 2 n \\ 12 \cdots 2 n\end{array}\right|$ is axisymmetric then ${ }_{\nu}^{\mu}={ }_{\mu}^{\nu}$ and the right-hand side of theorem $A$ vanishes, giving KronECKER's relation. If the determinant is centrosymmetric then the right-hand side of theorem $B$ vanishes, giving the relation pointed out by MUIR in 1888. In this paper three theorems [(1), (2), (3), below] and two formulas [I, II, below] are developed. Theorems $A$ and $B$, which may be characterized by the notations $A(n, n-1)$ and $B(n, 1)$ respectively, are extended, giving theorems (1) and (3), characterized by the notations $A(n, k)$ and $B(n, k),(1 \equiv k \equiv n-1)$, respectively. If we call the left-hand side of a Kronecker relation a Kronecker expression, then theorem (2) expresses a sum of Kronecker expressions as a sum of products of sums of Kronecker expressions of lower order. Formula I gives for a centrosymmetric determinant the number of true vanishing aggregates of each type, and formula II gives the number of those which are linearly independent. Part I (arts. 3-7) contains the work relating to theorem $A$ and Part II (arts. 8-11) contains the work relating to theorem $B$.

2. If we are given any combination (selection) of $2 n$ numbers $k$ at a time, the combination of the remaining $2 n-k$ numbers is said to be the complementary with respect to $2 n$ of the given combination. Let us use here, as elsewhere,*

* Cf. American Journal of Mathematics, vol. 22, no. 1. 
$(2 n \mid k) *$ to denote the $a$ th combination $k$ at a time of the $2 n$ numbers $1,2, \ldots$,

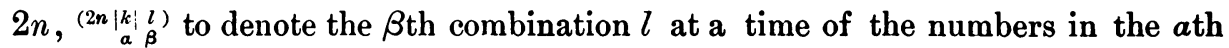
combination $k$ at a time of the $2 n$ numbers, and let $\left.{ }_{a}^{(2 \bar{n}} \mid k\right)$ denote the comple-

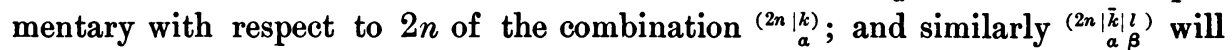
denote the complementary with respect to $k$ of the combination ${ }^{(2 n|k| l \mid l} \underset{a \beta}{ }$, i. e., the combination formed by the numbers remaining after the numbers in the combination ${ }^{(2 n|k| l)} \underset{a}{|c| l}$ are taken from the combination ${ }^{(2 n \mid k)}{ }_{a}$. The symbol ${ }^{(\overline{2 n}|\bar{k}| l)}$ will denote the complementary of $(\underset{2 n}{2 n}|k| l)$ with respect to $k$, i. e., the combination formed by the $2 n-k-l$ numbers remaining after the $l$ numbers in the combination $\underset{a \beta}{(2 n|k| l)}$ are taken from the combination $(\overline{2 n} \mid k)$. Similarly $(\overline{2 n}|\bar{k}| \bar{l} \mid h \ldots)$ is the complementary of $(\overline{2 n}|\bar{k}| l \mid h \ldots)$ with respect to $l$.

If $(a),(b)$ denote combinations of distinct numbers the notations $(a \gamma b)$ and $(a)(b)$ denote the combination of all the numbers involved in $(a)$ and $(b)$ taken respectively in loco or in natural order according as the brackets are or are not interlocked.

The notation $s_{t}$ will be used for $s ! / t !(s-t) !$

\section{Part I.}

3. In this part of the paper expressions occur containing minors and products of minors of the following form: $\mid\left[\begin{array}{l}\left(x_{1}\right) \\ \left.x_{2}\right)\end{array}||,\left[\begin{array}{l}y_{1} \\ \left(y_{2}\right)\end{array}|\cdot| \begin{array}{l}\left(z_{2}\right) \\ z_{2}\end{array}\right) \mid\right.$, where $\left.\left(x_{1}\right\rangle x_{2}\right)$ and $\left.\left(y_{1}\right\rangle z_{1} \backslash y_{2} \backslash z_{2}\right)$ are permutations of the $2 n$ numbers $1,2, \ldots, 2 n$. In general the sign factor for any term $\left|\begin{array}{l}\left(x_{1}\right) \\ \left(x_{2}\right)\end{array}\right| \cdot\left|\begin{array}{l}\left(y_{1}\right) \\ \left(y_{2}\right)\end{array}\right| \cdot\left|\begin{array}{l}\left.z_{1}\right) \\ \left(z_{2}\right)\end{array}\right| \cdots$ is $(-1)^{\nu}$ where $\nu$ is the number of inversions of order in $\left.\left.\left.\left(x_{1}\right\rangle y_{1}\right\rangle z_{1}\right) \cdots\left(x_{2} \backslash y_{2}\right\rangle z_{2}\right) \cdots$

With respect to a parent determinant of order $2 n$ the sum of minors of order $n$

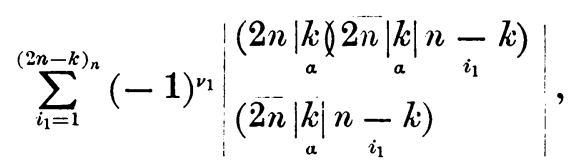

which has $k$ rows the same throughout may obviously be written in the form

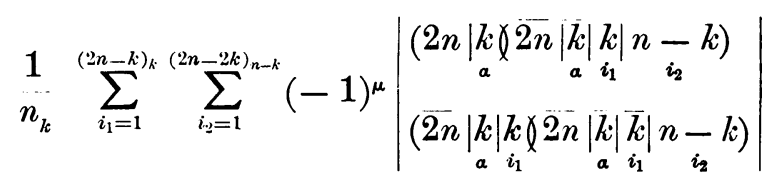

which, on expanding each term by Laplace's theorem in terms of minors formed from the ath selection of $k$ rows with their complementaries, and finally collecting all the terms containing

$$
\left|\begin{array}{l}
(2 n \mid \underset{a}{k}) \\
\left(2 n|\underset{a}{k}| \underset{i_{1}}{k}\right)
\end{array}\right|
$$

* Unless otherwise stated, it is understood that the numbers in a combination are arranged in their natural order (order of magnitude). 
as a factor, takes the form

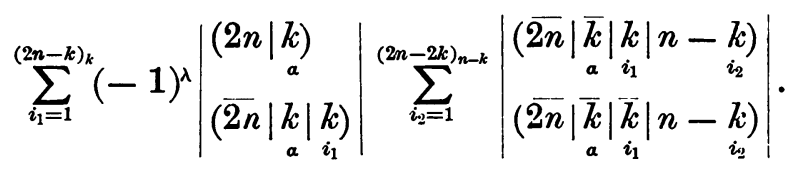

On examining the inner sum of this form one will easily see that any given selection of $h, 1 \equiv h \bar{\Sigma} n-k$, of the $2 n-2 k$ numbers in the combination

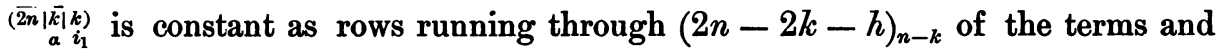
constant as columns through as many more terms. We therefore arrive at the following :

Theorem $(1)$ or $A(n, k)$ :

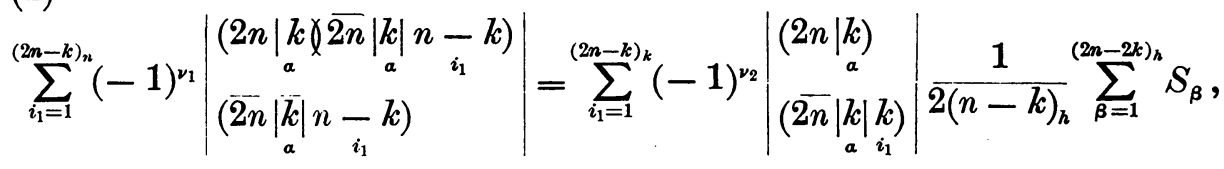

where $S_{\beta}$ denotes the sum of the two aggregates:

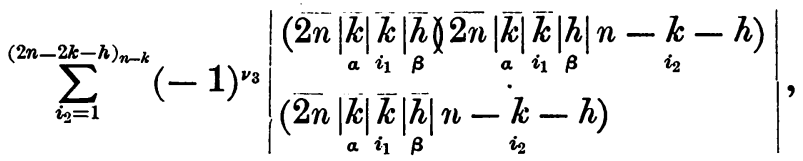

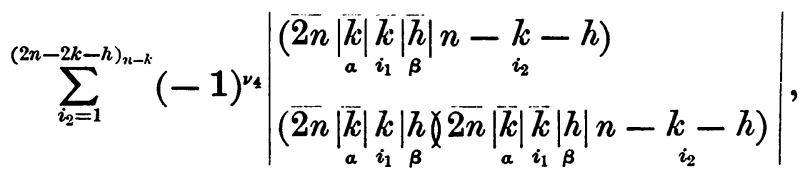

in which, if

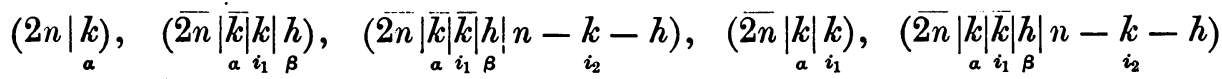
are denoted by

$$
(a),(b),(c),(d),(e)
$$

respectively, the integers $\nu_{2}, \nu_{3}, \nu_{4}$ denote the numbers of inversions in

respectively.

$$
\left(a_{\ell} b_{\ell} c_{\ell} d_{\ell} e\right),\left(b_{\ell} c_{\ell} e\right),\left(e_{\ell} b_{\ell} c\right)
$$

The identity of this theorem (1) or $A(n, k)$ depends upon any combination $\underset{a}{(2 n \mid k)}$ and any integer $h(1 \leqq h \leqq n-k)$.

For $h=1$ and $k=n-1$ theorem (1) reduces to Muir's theorem $A$. 
As an illustration of $(1)$ we have for $(n, k, h)=(4,2,1)$ :

$$
\begin{aligned}
\left|\begin{array}{l}
1234 \\
5678
\end{array}\right| & -\left|\begin{array}{l}
1235 \\
4678
\end{array}\right|+\left|\begin{array}{l}
1236 \\
4578
\end{array}\right|-\left|\begin{array}{l}
1237 \\
4568
\end{array}\right|+\left|\begin{array}{l}
1238 \\
4567
\end{array}\right|+\left|\begin{array}{l}
1245 \\
3678
\end{array}\right|-\left|\begin{array}{l}
1246 \\
3578
\end{array}\right|+\left|\begin{array}{l}
1247 \\
3568
\end{array}\right| \\
& -\left|\begin{array}{l}
1248 \\
3567
\end{array}\right|+\left|\begin{array}{l}
1256 \\
3478
\end{array}\right|-\left|\begin{array}{l}
1257 \\
3468
\end{array}\right|+\left|\begin{array}{l}
1258 \\
3467
\end{array}\right|+\left|\begin{array}{l}
1267 \\
3458
\end{array}\right|-\left|\begin{array}{l}
1268 \\
3457
\end{array}\right|+\left|\begin{array}{l}
1278 \\
3456
\end{array}\right| \\
& =\left|\begin{array}{l}
12 \\
34
\end{array}\right|\left\{\left|\begin{array}{l}
56 \\
78
\end{array}\right|-\left|\begin{array}{l}
57 \\
68
\end{array}\right|+\left|\begin{array}{l}
58 \\
67
\end{array}\right|+\left|\begin{array}{l}
67 \\
58
\end{array}\right|-\left|\begin{array}{l}
68 \\
57
\end{array}\right|+\left|\begin{array}{l}
78 \\
56
\end{array}\right|\right\} \\
& -\left|\begin{array}{l}
12 \\
35
\end{array}\right|\left\{\left|\begin{array}{l}
46 \\
78
\end{array}\right|-\left|\begin{array}{l}
47 \\
68
\end{array}\right|+\left|\begin{array}{l}
48 \\
67
\end{array}\right|+\left|\begin{array}{l}
67 \\
48
\end{array}\right|-\left|\begin{array}{l}
68 \\
47
\end{array}\right|+\left|\begin{array}{l}
78 \\
46
\end{array}\right|\right\} \\
& + \text { etc. } \\
& +\left|\begin{array}{l}
12 \\
78
\end{array}\right|\left\{\left|\begin{array}{l}
34 \\
56
\end{array}\right|-\left|\begin{array}{l}
35 \\
46
\end{array}\right|+\left|\begin{array}{l}
36 \\
45
\end{array}\right|+\left|\begin{array}{l}
45 \\
36
\end{array}\right|-\left|\begin{array}{l}
46 \\
35
\end{array}\right|+\left|\begin{array}{l}
56 \\
34
\end{array}\right|\right\} .
\end{aligned}
$$

4. The conjugate of every minor in the first aggregate of $S_{\beta}$ in (1) is found in the second with the same or opposite sign according as $n-k$ is even or odd. This is readily seen on observing that in bringing $\left.(b\rangle_{\ell} \chi_{\ell} e\right)$ to the form $\left(e{ }_{l} b_{\ell} c\right)$ the $n-k$ numbers in $(e)$ are carried over the $n-k$ numbers in $(c)$ and $(b)$ and therefore the exponents of the sign factors for the two differ, mod. 2, by $(n-\dot{k})^{2}$ or $n-k .^{*}$

5. Since each aggregate of $S_{\beta}$ on the right of $(1)$ is an expression involving minors of order $n-k$ the same in form as those on the left-hand side of the equation we may, if we choose, apply the theorem to them. If $n-k$ is not odd we may select $h$ so that, after applying the theorem a second time, $n-k-h$ is odd and shall have within each of the resulting $S_{\beta}$ 's conjugate minors with opposite signs.

6 . If now we take the sum

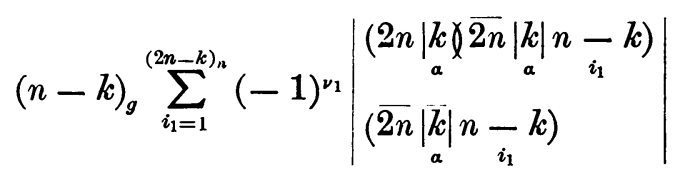

and, if instead of expanding each minor in terms of minors formed from the ath selection of $k$ rows as in art. 3, we expand in terms of minor formed from the $\beta$ th selection of $h$ of the ath selection of $k$ rows and $g$ others and finally collect like terms we get the following:

TheOREM (2):

$$
(n-k)_{g} \sum_{i_{1}=1}^{(2 n-k)_{n}}(-1)^{\nu_{1}}\left|\begin{array}{c}
\left(2 n\left|k \times \frac{k}{2 n}\right| k \mid n-k\right) \\
(\underset{a}{2 n}|\bar{k}| n-k) \\
a
\end{array}\right|=\sum_{i_{1}=1}^{(2 n-k)_{n+2 g}}(-1)^{\nu_{2}} P_{i_{1}},
$$

* Cf. American Journal of Mathematics, vol. 22, no. 1, art. 2, p. 56. 
where $P_{i_{1}}$ denotes the product of the two aggregates :

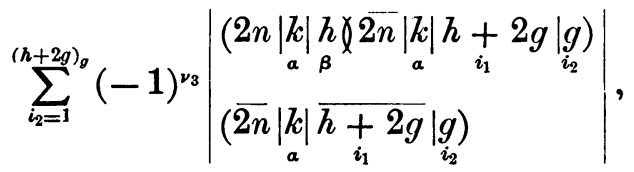

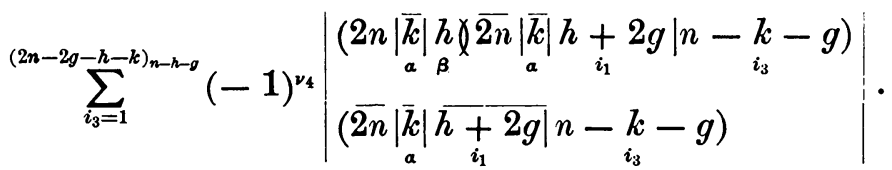

If $h=k$ and $g=1$, then (2) reduces to (1).

As an example of $(2)$ for $(n, k, g, h)=(3,1,1,1)$ we have

$$
\begin{aligned}
& 2\left\{\left|\begin{array}{l}
123 \\
456
\end{array}\right|-\left|\begin{array}{l}
124 \\
356
\end{array}\right|+\left|\begin{array}{l}
125 \\
346
\end{array}\right|-\left|\begin{array}{l}
126 \\
345
\end{array}\right|+\left|\begin{array}{l}
134 \\
256
\end{array}\right|-\left|\begin{array}{l}
135 \\
246
\end{array}\right|+\left|\begin{array}{l}
136 \\
245
\end{array}\right|+\left|\begin{array}{l}
145 \\
236
\end{array}\right|-\left|\begin{array}{l}
146 \\
235
\end{array}\right|+\left|\begin{array}{l}
156 \\
234
\end{array}\right|\right\} \\
& =\left(\begin{array}{l}
2 \\
3
\end{array}-\begin{array}{l}
3 \\
2
\end{array}\right)\left\{\left|\begin{array}{l}
14 \\
56
\end{array}\right|-\left|\begin{array}{c}
15 \\
46
\end{array}\right|+\left|\begin{array}{c}
16 \\
45
\end{array}\right|\right\}-\left(\begin{array}{c}
2 \\
4
\end{array}-\begin{array}{c}
4 \\
2
\end{array}\right)\left\{\left|\begin{array}{l}
13 \\
56
\end{array}\right||-| \begin{array}{l}
15 \\
36
\end{array}|+| \begin{array}{l}
16 \\
35
\end{array} \mid\right\} \\
& +\left(\begin{array}{l}
2 \\
5
\end{array}-\begin{array}{l}
5 \\
2
\end{array}\right)\left\{\left|\begin{array}{l}
13 \\
46
\end{array}\right|-\left|\begin{array}{l}
14 \\
36
\end{array}\right|+\left|\begin{array}{l}
16 \\
34
\end{array}\right|\right\}-\left(\begin{array}{ll}
2 & 6 \\
6 & 6
\end{array}\right)\left\{\left|\begin{array}{l}
13 \\
45
\end{array}\right|-\left|\begin{array}{l}
14 \\
35
\end{array}\right|+\left|\begin{array}{l}
15 \\
34
\end{array}\right|\right\} \\
& +\left(\begin{array}{cc}
3 & 4 \\
4 & 3
\end{array}\right)\left\{\left|\begin{array}{l}
12 \\
56
\end{array}\right|-\left|\begin{array}{l}
15 \\
26
\end{array}\right|+\left|\begin{array}{c}
16 \\
25
\end{array}\right|\right\}-\left(\begin{array}{cc}
3 & 5 \\
5 & 3
\end{array}\right)\left\{\left|\begin{array}{c}
12 \\
46
\end{array}\right|-\left|\begin{array}{c}
14 \\
26
\end{array}\right|+\left|\begin{array}{c}
16 \\
24
\end{array}\right|\right\}
\end{aligned}
$$

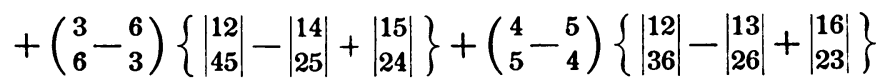

$$
\begin{aligned}
& -\left(\begin{array}{l}
4 \\
6
\end{array}-\begin{array}{l}
6 \\
4
\end{array}\right)\left\{\left|\begin{array}{l}
12 \\
35
\end{array}\right|-\left|\begin{array}{l}
13 \\
25
\end{array}\right|+\left|\begin{array}{l}
15 \\
23
\end{array}\right|\right\}+\left(\begin{array}{cc}
5 & 6 \\
6 & 3
\end{array}\right)\left\{\left|\begin{array}{l}
12 \\
34
\end{array}\right|-\left|\begin{array}{l}
13 \\
24
\end{array}\right|+\left|\begin{array}{c}
14 \\
23
\end{array}\right|\right\} \text {. }
\end{aligned}
$$

7. If now we impose the conditions for axisymmetry the right-hand side of (1) becomes zero and for $k=n-1$ is the general Kronecker relation. Under the same conditions of axisymmetry the left-hand side of (2), which in general we shall denote by $(n-k)_{g} K(n, k)$, is a sum of Kronecker expressions and the theorem (2) may be written schematically

$$
(n-k)_{g} K(n, k)=\sum K(h+g, h) K(n-g-h, k-h) .
$$

If $g=1$, then $K(h+g, h)$ is a Kronecker expression and if in addition $k=n-2$, then $K(n-g-h, k-h)$ is also a Kronecker expression, so that under these conditions (2) expresses a sum of Kronecker expressions for minors of order $n$ as a sum of products of Kronecker expressions for minors of orders $h+g$ and $n-h-g$ respectively. It is apparent that, by repeated applications of the theorem and by a proper choice of $g$, we may reduce both factors of each term on the right of (2) to Kronecker expressions.

If the original determinant is skew then the two aggregates of $S_{\beta}$ in (1) are equal. 


\section{Part II.}

8. If $(m)$ denotes any combination of the $2 n$ numbers $1,2, \ldots, 2 n$, then $(\bar{m})$ shall denote the combination formed by replacing every number in $(m)$ by its defect from $2 n+1$.

The extension of theorem B is now easily stated as follows:

TheOREM (3) or $B(n, k)$ :

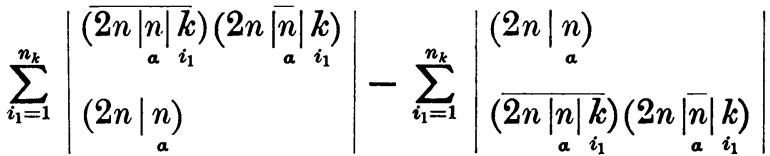

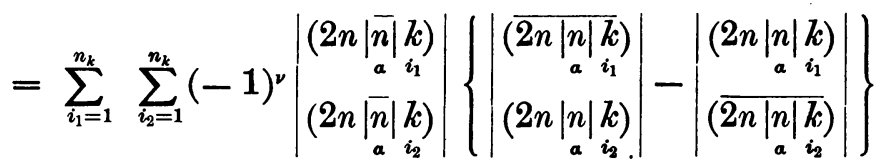

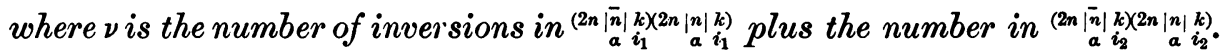

The proof of this theorem consists in the expansion by LAPLACE's theorem of each minor contained in the first sum on the left in terms of minors formed from the $n-k$ rows having for indices the numbers in $\begin{gathered}(2 n|\overline{\mid}| k) \\ a i_{1}\end{gathered}$, with their complementaries; and of each minor in the second sum on the left in terms of minors from the $n-k$ columns having the same numbers for column indices, with their complementaries, and finally in the collection of the terms in pairs one from each sum. Thus the left-hand side of (3) is equal to

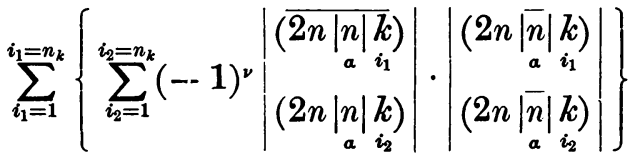

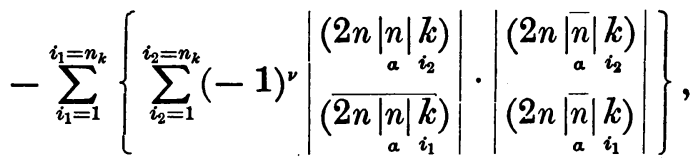

which in turn is equal to the right-hand side.

When $k=1$ the theorem $B(n, k)$ reduces to MuIR's theorem $B$.

9. Under the condition for centrosymmetry the right-hand side of (3) vanishes and we have the general linear relation connecting minors of order $n$ of a centrosymmetric determinant of order $2 n$. The type $k=1$ is that which was first pointed out by MUIR in 1888.

10. MuIr in his paper of 1900 states in connection with the identities of the type $k=1$ given by him; "that the number of such identities possible, when the centrosymmetry is complete and the parent determinant is of the $2 n$th order, 
is $C_{2 n, n}$." It is to be observed, however, that we do not get a true vanishing aggregate for every selection of the $2 n$ numbers $n$ at a time. For if $x$ and $y$ are any two of the $n$ numbers in any given selection, then whenever $x+y=2 n+1$ some of the minors in the first sum have their conjugates in the second and cancel each other, while others will vanish identically. Those that remain, if any. form an aggregate which is the extensional of a vanishing aggregate of minors of lower order.

Thus for a determinant of order six if we take the selection 256 we get

$$
\left|\begin{array}{l}
251 \\
256
\end{array}\right|+\left|\begin{array}{l}
226 \\
256
\end{array}\right|+\left|\begin{array}{l}
556 \\
256
\end{array}\right|-\left|\begin{array}{l}
256 \\
251
\end{array}\right|-\left|\begin{array}{l}
256 \\
226
\end{array}\right|-\left|\begin{array}{l}
256 \\
556
\end{array}\right|,
$$

in which the first and fourth are conjugate and therefore cancel, the others are indentically zero having identical rows or columns.

For a determinant of order eight, taking the selection 1678 we get

$$
\left|\begin{array}{l}
1671 \\
1678
\end{array}\right|+\left|\begin{array}{l}
1628 \\
1678
\end{array}\right|+\left|\begin{array}{l}
1378 \\
1678
\end{array}\right|+\left|\begin{array}{l}
8678 \\
1678
\end{array}\right|-\left|\begin{array}{c}
1678 \\
1671
\end{array}\right|-\left|\begin{array}{c}
1678 \\
1628
\end{array}\right|-\left|\begin{array}{l}
1678 \\
1378
\end{array}\right|-\left|\begin{array}{l}
1678 \\
8678
\end{array}\right|
$$

which reduces to the extensional of

$$
\left|\begin{array}{l}
62 \\
67
\end{array}\right|+\left|\begin{array}{l}
37 \\
67
\end{array}\right|-\left|\begin{array}{l}
67 \\
62
\end{array}\right|-\left|\begin{array}{l}
67 \\
37
\end{array}\right| .
$$

Neglecting these degenerate aggregates we have, if $\lambda$ denotes the number of true vanishing aggregates of each type (i. e., for each of the $n-1$ values of $k$ ), for the determination of $\lambda$ the following:

Formula I :

$$
\lambda=\frac{1}{2}\left[C_{2 n, n}-\sum_{r=1}^{r=[n / 2]} \frac{n(n-1) \cdots(n-2 r+1)}{(r !)^{2}} 2^{n-2 r}\right] .
$$

This we see by counting the number of combinations containing one, two, three, etc., pairs of numbers satisfying the equation $x+y=2 n+1$, and subtracting the result from $C_{2 n, n}$ and observing that for every relation

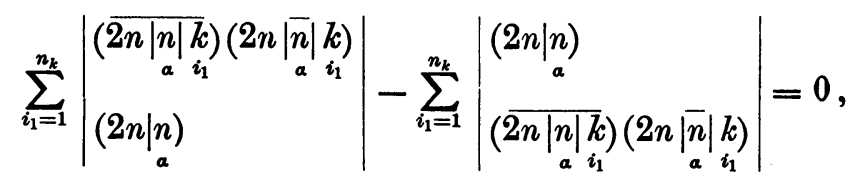

there is another

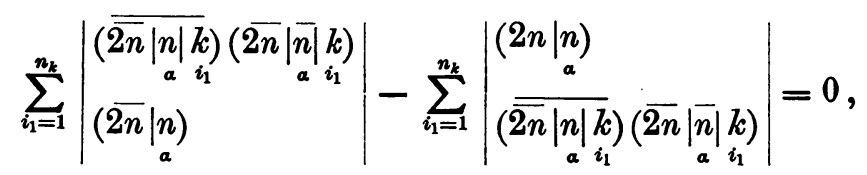

which in virtue of the centrosymmetry of the determinant is not different from the first. 
11. On examining a set of $\lambda$ true vanishing aggregates of any type $k$ it will be seen that:

(a) Each aggregate contains $n_{k}$ terms together with the negative of their conjugates.

(b) Every minor $\left.\mid \begin{array}{l}(a) \\ b\end{array}\right) \mid$ enters two aggregates, in one with $(b)$ as the selection of columns and in another with $(a)$, (or $(\bar{a}))$, as the selection of columns.

(c) There are $n_{k} \lambda$ independent minors $\left(\frac{1}{2} n_{k} \lambda\right.$ with their conjugates).

(d) For every set of $\frac{1}{2} \lambda$ aggregates there is a set of $\frac{1}{2} \lambda$ minors (one in each aggregate) whose conjugates are found in the remaining $\frac{1}{2} \lambda$ aggregates.

$(e)$ The sum of the $\lambda$ aggregates vanishes.

It follows from $(a)$ and $(b)$ that every minor enters one aggregate with the positive and one with the negative sign, and that any two aggregates have either two or no minors in common.

It follows from $(d)$ that the sum of no fewer than $\lambda$ aggegates can vanish.

If we denote the number of linearly independent aggregates of each type by $\mu$ then we have for the determination of $\mu$ the following:

Formula II :

$$
\mu=\lambda-1 \text {. }
$$

SyraCuse University, March 15, 1901. 